\title{
Canonical Kernels Versus Constructible Kernels萌
}

\author{
Steven G. Krantz \\ September 13, 2018
}

\begin{abstract}
We study both canonical reproducing kernels and constructive reproducing kernels for holomorphic functions in $\mathbb{C}^{1}$ and $\mathbb{C}^{n}$. We compare and contrast the two, and also develop important relations between the two types of kernels. We prove a new result about the relationship between these two kernels on certain domains of finite type.
\end{abstract}

\section{Introduction}

Every working mathematician has encountered integral reproducing formulas for holomorphic functions. The Cauchy integral formula and the Poisson integral formula are perhaps the two most central and important examples. These are examples of constructive reproducing formulas (kernels) because the integral formulas (kernels) can often be written down explicitly or perhaps asymptotically (see [KRA2] and [KRA4]). What is of interest for our purpose here is that there are other integral reproducing formulas, which are canonical in nature (to be explained below), but for which the formulas (kernels) generally cannot be written down explicitly. Often the canonical kernels have many attractive features, but the fact that they are not explicit means that we do not necessarily understand their singularities, and therefore it is difficult to analyze them or to make estimates on them. We find it nearly impossible to determine their mapping properties.

But there are techniques for making peace between the canonical and the constructive. And these methods can be extremely useful in practice. The purpose of this paper is to explain these connections, and to explore where they might lead. Furthermore, we shall prove a version of the Kerzman/Stein theorem relating canonical and constructible kernels on strongly pseudoconvex domains in the more general context of convex, finite type domains.

\footnotetext{
${ }^{1}$ Key Words: canonical kernel, constructible kernel, Cauchy kernel, Bergman kernel, Szegő kernel

${ }^{2}$ MR Classification Numbers: 30C40, 32A25, 32A26, 46E22, 47B32
} 


\section{First Principles}

In what follows, a domain is a connected open set.

It is arguable that many, if not most, constructible integral reproducing formulas are consequences of (and often equivalent to) the fundamental theorem of calculus. Or, in several variables, we would substitute "Stokes's theorem" for "fundamental theorem of calculus." As a simple example,

$$
f(x)-f(a)=\int_{a}^{x} f^{\prime}(t) d t
$$

is an integral reproducing formula. If $\varphi$ is a $C^{1}$ function on $[a, x]$ such that $\varphi(a)=0$ and $\varphi(x)=1$ then we have (from Leibniz's product rule)

$$
f(x)=\int_{a}^{x} f(t) \varphi^{\prime}(t) d t+\int_{a}^{x} f^{\prime}(t) \varphi(t) d t .
$$

In this way we have introduced the kernels $\varphi$ and $\varphi^{\prime}$. The monograph [BIN, pp. 103-113] contains incisive generalizations of this last reproducing formula to the several-variable context. It is again worth noting that all these formulas are based on the fundamental theorem of calculus (or Stokes's theorem).

A perhaps more profound illustration of the connection between Stokes's theorem and an important and central integral reproducing formula is the generalized Cauchy integral formula. We begin with a key but ancillary idea.

Lemma 1.1 Let $\Omega$ be a bounded domain in $\mathbb{C}$ with $C^{1}$ boundary. Let $\omega=$ $\alpha(z) d z$ be a 1 -form with coefficient that is continuously differentiable on $\bar{\Omega}$. Then

$$
\int_{\partial \Omega} \omega=\iint_{\Omega} \frac{\partial \alpha}{\partial \bar{z}} d \bar{z} \wedge d z
$$

For a general 1-form $\lambda=a(z) d z+b(z) d \bar{z}$, it is useful to write

$$
\begin{aligned}
d \lambda & =\partial \lambda+\bar{\partial} \lambda \\
& =\left[\frac{\partial a}{\partial z} d z \wedge d z+\frac{\partial b}{\partial z} d z \wedge d \bar{z}\right]+\left[\frac{\partial a}{\partial \bar{z}} d \bar{z} \wedge d z+\frac{\partial b}{\partial \bar{z}} d \bar{z} \wedge d \bar{z}\right] \\
& =\frac{\partial a}{\partial \bar{z}} d \bar{z} \wedge d z+\frac{\partial b}{\partial z} d z \wedge d \bar{z}
\end{aligned}
$$

Recall that, for a $C^{1}$ function $f$,

$$
\frac{\partial}{\partial z} f=\frac{1}{2}\left(\frac{\partial}{\partial x}-i \frac{\partial}{\partial y}\right) f \quad \text { and } \quad \frac{\partial}{\partial \bar{z}} f=\frac{1}{2}\left(\frac{\partial}{\partial x}+i \frac{\partial}{\partial y}\right) f .
$$

It is convenient to also introduce here the notation

$$
\partial f=\frac{\partial f}{\partial z} d z \quad \text { and } \quad \bar{\partial} f=\frac{\partial f}{\partial \bar{z}} d \bar{z} .
$$


There is complex exterior differential notation for forms, and we refer the reader to [KRA2] or [WEL] for details of that topic.

With this notation, formula (1.1.1) in the theorem becomes

$$
\int_{\partial \Omega} \omega=\iint_{\Omega} \bar{\partial} \omega
$$

Now we prove a generalized version of the Cauchy integral formula. Note that it is valid for essentially all functions - not just holomorphic functions.

Theorem 1.2 If $\Omega \beta \mathbb{C}$ is a bounded domain with $C^{1}$ boundary and if $f \in C^{1}(\bar{\Omega})$ then, for any $z \in \Omega$,

$$
f(z)=\frac{1}{2 \pi i} \int_{\partial \Omega} \frac{f(\zeta)}{\zeta-z} d \zeta-\frac{1}{2 \pi i} \iint_{\Omega} \frac{(\partial f(\zeta) / \partial \bar{\zeta})}{\zeta-z} d \bar{\zeta} \wedge d \zeta .
$$

Proof: Fix $z \in \Omega$ and choose $\epsilon>0$ so that $\bar{D}(z, \epsilon) B \Omega$. Set $\Omega_{\epsilon}=\Omega \backslash \bar{D}(z, \epsilon)$. We apply Stokes's theorem to the form

$$
\omega(\zeta)=\frac{f(\zeta) d \zeta}{\zeta-z}
$$

on the domain $\Omega_{\epsilon}$. Note here that $\omega$ has no singularity on $\overline{\Omega_{\epsilon}}$.

Thus

$$
\int_{\partial \Omega_{\epsilon}} \omega(\zeta)=\iint_{\Omega_{\epsilon}} \bar{\partial} \omega
$$

Writing this out gives

$$
\int_{\partial \Omega} \frac{f(\zeta)}{\zeta-z} d \zeta-\int_{\partial D(z, \epsilon)} \frac{f(\zeta)}{\zeta-z} d \zeta=\iint_{\Omega_{\epsilon}} \frac{\partial f(\zeta) / \partial \bar{\zeta}}{\zeta-z} d \bar{\zeta} \wedge d \zeta
$$

Observe that we have reversed the orientation in the second integral on the left because the disk is inside the region $\Omega$.

Now, as $\epsilon \rightarrow 0^{+}$, the integral on the right tends to

$$
\iint_{\Omega} \frac{\partial f(\zeta) / \partial \bar{\zeta}}{\zeta-z} d \bar{\zeta} \wedge d \zeta
$$

[We use here the fact that $1 /(\zeta-z)$ is integrable.] The first integral on the left does not depend on $\epsilon$. The second integral on the left requires a little analysis:

$$
\int_{\partial D(z, \epsilon)} \frac{f(\zeta)}{\zeta-z} d \zeta=\int_{0}^{2 \pi} \frac{f\left(z+\epsilon e^{i t}\right)}{\epsilon e^{i t}} \cdot i \epsilon e^{i t} d t=i \int_{0}^{2 \pi} f\left(z+\epsilon e^{i t}\right) d t .
$$

Now the last expression tends, as $\epsilon \rightarrow 0^{+}$, to $2 \pi i f(z)$. 
Putting all this information into equation (1.2.1) yields

$$
f(z)=\frac{1}{2 \pi i} \int_{\partial \Omega} \frac{f(\zeta)}{\zeta-z} d \zeta-\frac{1}{2 \pi i} \iint_{\Omega} \frac{\partial f / \partial \bar{\zeta}}{\zeta-z} d \bar{\zeta} \wedge d \zeta
$$

as desired.

Corollary 1.3 With hypotheses as in Theorem 1.2, and the additional assumption that $\bar{\partial} f=0$ on $\Omega$, we have

$$
f(z)=\frac{1}{2 \pi i} \int_{\partial \Omega} \frac{f(\zeta)}{\zeta-z} d \zeta
$$

Remark 1.4 Corollary 1.3 is the familiar Cauchy integral formula from the analysis of one complex variable.

Thus we have seen Stokes's theorem, in its complex formulation, used in an important fashion to derive a decisive integral formula.

\section{Canonical Integral Formulas}

One of the first canonical integral formulas ever created was that of Stefan Bergman [BER]. We shall present Bergman's idea in the context of a more general construction due to Nachman Aronszajn [ARO]. This is the idea of a Hilbert space with reproducing kernel. Fortunately Aronszajn's idea also entails the Szegö kernel and several other important reproducing kernels.

Definition 2.1 Let $X$ be any set and let $\mathcal{H}$ be a Hilbert space of complexvalued functions on $X$. We say that $\mathcal{H}$ is a Hilbert space with reproducing kernel if, for each $x \in X$, the linear (point evaluation) map of the form

$$
\begin{aligned}
L_{x}: \mathcal{H} & \longrightarrow \mathbb{C} \\
f & \longmapsto f(x),
\end{aligned}
$$

is continuous. We write this as

$$
|f(x)| \leq C \cdot\|f\|_{\mathcal{H}} .
$$

In this circumstance, the classical Riesz representation theorem (see [RUD]) tells us that, for each $x \in X$, there is a unique element $k_{x} \in \mathcal{H}$ such that

$$
f(x)=\left\langle f, k_{x}\right\rangle \quad \forall f \in \mathcal{H} .
$$

We then define a function

$$
K: X \times X \rightarrow \mathbb{C}
$$

by the formula

$$
K(x, y) \equiv \overline{k_{x}(y)} .
$$

The function $K$ is the reproducing kernel for the Hilbert space $\mathcal{H}$. 
We see that $K$ is uniquely determined by $\mathcal{H}$ because, again by the Riesz representation theorem, the element $k_{x}$ for each $x \in \mathcal{H}$ is unique.

It is a classical fact, and we shall not provide the details here (but see [KRA2] for all the particulars), that the kernel $K$ may be (at least in principle) constructed by way of a complete orthonormal basis for $\mathcal{H}$. To wit, let $\left\{\varphi_{j}\right\}$ be such a basis. Then

$$
K(x, y)=\sum_{j=1}^{\infty} \varphi_{j}(x) \overline{\varphi_{j}(y)} .
$$

Here the convergence is in the Hilbert space topology in each variables. And in fact the fundamental property (2.1.1) of a Hilbert space with reproducing kernel shows that the convergence is uniform on compact subsets of $X \times X$.

In what follows, on $\mathbb{C}^{n}$, we use $d V$ to denote volume measure.

EXAMPLE 2.2 Let $\Omega$ be a bounded domain in $\mathbb{C}$ or $\mathbb{C}^{n}$. Define

$$
A^{2}(\Omega)=\left\{f \text { holomorphic on } \Omega: \int_{\Omega}|f(z)|^{2} d V(z)<\infty\right\} .
$$

Clearly $A^{2}(\Omega)$ is a complex linear space. It is commonly called the Bergman space. We equip $A^{2}(\Omega)$ with the norm $\|f\|_{A^{2}(\Omega)} \equiv\left[\int_{\Omega}|f|^{2} d V\right]^{1 / 2}$.

We have the following important preliminary result.

Lemma 2.3 There is a constant $C=C(K, \Omega)$, depending only on the domain $\Omega$ and on $K$ compact in $\Omega$, such that, if $f \in A^{2}(\Omega)$, then

$$
\sup _{z \in K}|f(z)| \leq C\left(\int_{\Omega}|f(\zeta)|^{2} d V(\zeta)\right)^{1 / 2} \equiv C \cdot\|f\|_{A^{2}(\Omega)} .
$$

Proof: Choose $r>0$ so that, if $z \in K$, then $B(z, r) B \Omega$. Then, for $z \in K$, we have

$$
\begin{aligned}
|f(z)| & =\frac{1}{V(B(z, r))}\left|\int_{B(z, r)} f(\zeta) d V(\zeta)\right| \\
& \leq \frac{1}{\sqrt{V(B(z, r))}} \int_{B(z, r)}|f(\zeta)|^{2} d V(\zeta)^{1 / 2} \\
& \leq C(\Omega, K)\left(\int_{\Omega}|f(\zeta)|^{2} d V(\zeta)\right)^{1 / 2} \\
& \equiv C(\Omega, K) \cdot\|f\|_{A^{2}(\Omega)} .
\end{aligned}
$$

That proves the result. 
With the indicated norm, the space $A^{2}(\Omega$ is a Hilbert space. For the completeness, note that if $\left\{f_{j}\right\}$ is a Cauchy sequence, then it will converge in the $L^{2}$ topology to some limit function $g$. But the lemma tells us that, for holomorphic functions, $L^{2}$ convergence implies uniform convergence on compact sets (sometimes called normal convergence). Hence the limit function is holomorphic and $L^{2}$, thus a member of $A^{2}$.

Of course the lemma tells us immediately that $A^{2}(\Omega)$ is a Hilbert space with reproducing kernel (just take the compact set $K$ to be a singleton $\{z\}$ ). The kernel $K$ is known as the Bergman kernel. It is one of the most important invariants of modern function theory.

EXAMPLE 2.4 Using the remark preceding Example 2.2, it is possible to calculate the Bergman kernel for the disc. We do so now.

Let $\psi(\zeta)=\zeta^{j}$ on the disc, $j=0,1,2, \ldots$ The theory of power series tells us that these functions form a complete basis for $A^{2}(D)$. Moreover, by parity, these functions are pairwise orthogonal. A simple calculation with polar coordinates shows that

$$
\left\|\psi_{j}\right\|_{A^{2}(D)}=\sqrt{\pi /(j+1)} .
$$

Therefore

$$
\varphi_{j}(\zeta)=\sqrt{\frac{j+1}{\pi}} \zeta^{j}
$$

forms a complete orthonormal basis for $A^{2}(\Omega)$.

Using the formula preceding Example 2.2, we then see that

$$
K(z, \zeta)=\sum_{j=0}^{\infty} \frac{j+1}{\pi}(z \bar{\zeta})^{j} .
$$

Let $\alpha=z \bar{\zeta}$. So we have

$$
\sum_{j=0}^{\infty} \frac{j+1}{\pi} \alpha^{j}=\frac{1}{\pi} \frac{d}{d \alpha} \sum_{j=0}^{\infty} a^{j+1}=\frac{1}{\pi} \frac{d}{d \alpha} \alpha \sum_{j=0}^{\infty} a^{j}=\frac{1}{\pi} \frac{d}{d \alpha} \alpha \cdot \frac{1}{1-\alpha}=\frac{1}{\pi} \cdot \frac{1}{1-\alpha^{2}} .
$$

In conclusion,

$$
K(z, \zeta)=\frac{1}{\pi} \cdot \frac{1}{(1-z \bar{\zeta})^{2}} .
$$

EXAMPLE 2.5 We now want to describe the Szegő theory. In order to make this work, we need to present some preliminary results about the BochnerMartinelli kernel and integral representation formula.

Definition 2.6 On $\mathbb{C}^{n}$ we let

$$
\begin{aligned}
\omega(z) & \equiv d z_{1} \wedge d z_{2} \wedge \cdots \wedge d z_{n} \\
\eta(z) & \equiv \sum_{j=1}^{n}(-1)^{j+1} z_{j} d z_{1} \wedge \cdots \wedge d z_{j-1} \wedge d z_{j+1} \wedge \cdots \wedge d z_{n} .
\end{aligned}
$$


The form $\eta$ is sometimes called the Leray form. We shall often write $\omega(\bar{z})$ to mean $d \bar{z}_{1} \wedge \cdots \wedge d \bar{z}_{n}$ and likewise $\eta(\bar{z})$ to mean $\sum_{j=1}^{n}(-1)^{j+1} \bar{z}_{j} d \bar{z}_{1} \wedge \cdots \wedge d \bar{z}_{j-1} \wedge$ $d \bar{z}_{j+1} \wedge \cdots \wedge d \bar{z}_{n}$.

The genesis of the Leray form is explained by the following lemma.

Lemma 2.7 For any $z_{0} \in \mathbb{C}^{n}$, any $\epsilon>0$, we have

$$
\int_{\partial B\left(z^{0}, \epsilon\right)} \eta(\bar{z}) \wedge \omega(z)=n \int_{B\left(z^{0}, \epsilon\right)} \omega(\bar{z}) \wedge \omega(z) .
$$

Proof: Notice that $d \eta(\bar{z})=\bar{\partial} \eta(\bar{z})=n \omega(\bar{z})$. Therefore, by Stokes's theorem,

$$
\int_{\partial B\left(z^{0}, \epsilon\right)} \eta(\bar{z}) \wedge \omega(z)=\int_{B\left(z^{0}, \epsilon\right)} d[\eta(\bar{z}) \wedge \omega(z)] .
$$

Of course the expression in [ ] is saturated in $d z^{\prime}$ 's so, in the decomposition $d=\partial+\bar{\partial}$, only the term $\bar{\partial}$ will not die. Thus the last line equals

$$
\int_{B\left(z^{0}, \epsilon\right)}[\bar{\partial}(\eta(\bar{z}))] \wedge \omega(z)=n \int_{B\left(z^{0}, \epsilon\right)} \omega(\bar{z}) \wedge \omega(z) .
$$

Remark 2.8 Notice that, by change of variables,

$$
\begin{aligned}
\int_{B\left(z^{0}, \epsilon\right)} \omega(\bar{z}) \wedge \omega(z) & =\int_{B(0, \epsilon)} \omega(\bar{z}) \wedge \omega(z) \\
& =\epsilon^{2 n} \int_{B(0,1)} \omega(\bar{z}) \wedge \omega(z) .
\end{aligned}
$$

A straightforward calculation shows that

$$
\begin{aligned}
& \int_{B(0,1)} \omega(\bar{z}) \wedge \omega(z) \\
& \quad=(-1)^{q(n)} \cdot(2 i)^{n} \cdot\left(\text { volume of the unit ball in } \mathbb{C}^{n} \approx \mathbb{R}^{2 n}\right),
\end{aligned}
$$

where $q(n)=[n(n-1)] / 2$. We denote the value of this integral by $W(n)$.

Theorem 2.9 (Bochner-Martinelli) Let $\Omega \beta \mathbb{C}^{n}$ be a bounded domain with $C^{1}$ boundary. Let $f \in C^{1}(\bar{\Omega})$. Then, for any $z \in \Omega$, we have

$$
\begin{aligned}
f(z)= & \frac{1}{n W(n)} \int_{\partial \Omega} \frac{f(\zeta) \eta(\bar{\zeta}-\bar{z}) \wedge \omega(\zeta)}{|\zeta-z|^{2 n}} \\
& -\frac{1}{n W(n)} \int_{\Omega} \frac{\bar{\partial} f(\zeta)}{|\zeta-z|^{2 n}} \wedge \eta(\bar{\zeta}-\bar{z}) \wedge \omega(\zeta) .
\end{aligned}
$$

Proof: Fix $z \in \Omega$. We apply Stokes's theorem to the form

$$
M_{z}(\zeta) \equiv \frac{f(\zeta) \eta(\bar{\zeta}-\bar{z}) \wedge \omega(\zeta)}{|\zeta-z|^{2 n}}
$$


on the domain $\Omega_{z, \epsilon} \equiv \Omega \backslash \bar{B}(z, \epsilon)$, where $\epsilon>0$ is chosen so small that $\bar{B}(z, \epsilon) B \Omega$. Note that Stokes's theorem does not apply to forms that have a singularity; thus we may not apply the theorem to $L_{z}$ on any domain that contains the point $z$ in either its interior or its boundary. This observation helps to dictate the form of the domain $\Omega_{z, \epsilon}$. As the proof develops, we shall see that it also helps to determine the outcome of our calculation.

Notice that

$$
\partial\left(\Omega_{z, \epsilon}\right)=\partial \Omega \cup \partial B(z, \epsilon)
$$

but that the two pieces are equipped with opposite orientations.

Thus, by Stokes,

$$
\begin{aligned}
\int_{\partial \Omega} M_{z}(\zeta)-\int_{\partial B(z, \epsilon)} M_{z}(\zeta) & =\int_{\partial \Omega_{z, \epsilon}} M_{z}(\zeta) \\
& =\int_{\Omega_{z, \epsilon}} d_{\zeta}\left(M_{z}(\zeta)\right) .
\end{aligned}
$$

Notice that we consider $z$ to be fixed and $\zeta$ to be the variable. Now

$$
\begin{aligned}
d_{\zeta} M_{z}(\zeta)= & \bar{\partial}_{\zeta} M_{z}(\zeta) \\
= & \frac{\bar{\partial} f(\zeta) \wedge \eta(\bar{\zeta}-\bar{z}) \wedge \omega(\zeta)}{|\zeta-z|^{2 n}} \\
& +f(\zeta) \cdot\left[\sum_{j=1}^{n} \frac{\partial}{\partial \bar{\zeta}_{j}}\left(\frac{\bar{\zeta}_{j}-\bar{z}_{j}}{|\zeta-z|^{2 n}}\right)\right] \omega(\bar{\zeta}) \wedge \omega(\zeta)
\end{aligned}
$$

Observing that

$$
\frac{\partial}{\partial \bar{\zeta}_{j}}\left(\frac{\bar{\zeta}_{j}-\bar{z}_{j}}{|\zeta-z|^{2 n}}\right)=\frac{1}{|\zeta-z|^{2 n}}-n \frac{\left|\bar{\zeta}_{j}-\bar{z}_{j}\right|^{2}}{|\zeta-z|^{2 n+2}},
$$

we find that the second term on the far right of (2.9.2) dies and we have

$$
d_{\zeta} M_{z}(\zeta)=\frac{\bar{\partial} f(\zeta) \wedge \eta(\bar{\zeta}-\bar{z}) \wedge \omega(\zeta)}{|\zeta-z|^{2 n}} .
$$

Substituting this identity into (2.9.1) yields

$$
\int_{\partial \Omega} M_{z}(\zeta)-\int_{\partial B(z, \epsilon)} M_{z}(\zeta)=\int_{\Omega_{z, \epsilon}} \frac{\bar{\partial} f(\zeta) \wedge \eta(\bar{\zeta}-\bar{z}) \wedge \omega(\zeta)}{|\zeta-z|^{2 n}} .
$$


Next we remark that

$$
\begin{aligned}
\int_{\partial B(z, \epsilon)} M_{z}(\zeta) & =f(z) \int_{\partial B(z, \epsilon)} \frac{\eta(\bar{\zeta}-\bar{z}) \wedge \omega(\zeta)}{|\zeta-z|^{2 n}} \\
& +\int_{\partial B(z, \epsilon)} \frac{(f(\zeta)-f(z)) \eta(\bar{\zeta}-\bar{z}) \wedge \omega(\zeta)}{|\zeta-z|^{2 n}} \\
& \equiv T_{1}+T_{2} .
\end{aligned}
$$

Since $|f(\zeta)-f(z)| \leq C|\zeta-z|$ (and since each term of $\eta(\bar{\zeta}-\bar{z}$ ) has a factor of some $\left.\bar{\zeta}_{j}-\bar{z}_{j}\right)$, it follows that the integrand of $T_{2}$ is of size $O(|\zeta-z|)^{-2 n+2} \approx \epsilon^{-2 n+2}$. Since the surface over which the integration is performed has area $\approx \epsilon^{2 n-1}$, it follows that $T_{2} \rightarrow 0$ as $\epsilon \rightarrow 0^{+}$.

By Lemma 2.7 and the remark following, we also have

$$
\begin{aligned}
T_{1} & =\epsilon^{-2 n} f(z) \int_{\partial B(z, \epsilon)} \eta(\bar{\zeta}-\bar{z}) \wedge \omega(\zeta) \\
& =n \epsilon^{-2 n} f(z) \int_{B(0, \epsilon)} \omega(\bar{\zeta}) \wedge \omega(\zeta) \\
& =n W(n) f(z) .
\end{aligned}
$$

Finally, (2.9.3)-(2.9.5) yield that

$$
\left(\int_{\partial \Omega} M_{z}(\zeta)\right)-n W(n) f(z)+o(1)=\int_{\Omega_{z, \epsilon}} \bar{\partial} f(\zeta) \wedge\left[\frac{\eta(\bar{\zeta}-\bar{z})}{|\zeta-z|^{2 n}}\right] \wedge \omega(\zeta) .
$$

Since

$$
\left|\frac{\eta(\bar{\zeta}-\bar{z})}{|\zeta-z|^{2 n}}\right|=O\left(|\zeta-z|^{-2 n+1}\right)
$$

the last integral is absolutely convergent as $\epsilon \rightarrow 0^{+}$(remember that $\bar{\partial} f$ is bounded). Thus we finally have

$$
f(z)=\frac{1}{n W(n)} \int_{\partial \Omega} L_{z}(\zeta)-\frac{1}{n W(n)} \int_{\Omega} \bar{\partial} f(\zeta) \wedge \frac{\eta(\bar{\zeta}-\bar{z})}{|\zeta-z|^{2 n}} \wedge \omega(\zeta) .
$$

This is the Bochner-Martinelli formula.

Remark 2.10 We see that the Bochner-Martinelli formula is a quintessential example of a constructible integral formula. The kernel is quite explicit, and it is the same for all domains. For the Bergman kernel, and for other canonical kernels that we shall see below, this latter property does not hold.

We note that the classical Cauchy integral formula in one complex variable is an immediate consequence of our new Bochner-Martinelli formula. 
Corollary 2.11 If $\Omega \beta \mathbb{C}^{n}$ is bounded and has $C^{1}$ boundary and if $f \in C^{1}(\bar{\Omega})$ and $\bar{\partial} f=0$ on $\Omega$, then

$$
f(z)=\frac{1}{n W(n)} \int_{\partial \Omega} \frac{f(\zeta) \eta(\bar{\zeta}-\bar{z})}{|\zeta-z|^{2 n}} \wedge \omega(\zeta) .
$$

Corollary 2.12 In complex dimension 1, the last corollary says that

$$
f(z)=\frac{1}{2 \pi i} \oint_{\partial \Omega} \frac{f(\zeta)}{\zeta-z} d \zeta
$$

Corollary 2.11 is particularly interesting. Like the classical Cauchy formula, it gives a constructible integral reproducing formula that is the same on all domains. Unlike the classical Cauchy formula, its kernel is not holomorphic in the free variable $z$. This makes the Bochner-Martinelli formula of limited utility in constructing holomorphic functions.

We note that Corollary 2.11 holds for broader classes of holomorphic functionssuch as the Hardy classes. One sees this by a simple limiting argument. See our discussion of $H^{2}$ below.

Now we turn to the development of the Szegö theory. Let $\Omega$ be a bounded domain in $\mathbb{C}$ or $\mathbb{C}^{n}$ with $C^{1}$ boundary. Define $A(\Omega)$ to be those functions which are continuous on $\bar{\Omega}$ and holomorphic on $\Omega$. Identify each element of $A(\Omega)$ with its boundary trace. Define $H^{2}(\Omega)$ to be the closure in the $L^{2}(\partial \Omega)$ norm of $A(\Omega)$. If $z \in \Omega$ is fixed then, by inspection of the formula in Corollary 2.11, and the Schwarz inequality,

$$
|f(z)| \leq C \cdot\|f\|_{H^{2}(\Omega)} .
$$

Thus we see that $H^{2}(\Omega)$ is a Hilbert space with reproducing kernel. We denote the kernel for this space by $S(z, \zeta)$.

Using the comment preceding Example 2.2 of Section 2, we can actually calculate the Szegö kernel on the disc. We first note that $\left\{z^{j}\right\}_{j=0}^{\infty}$ forms a basis for the Hilbert space $H^{2}(D)$. This follows from the standard theory of power series for holomorphic functions on $D$. It is orthogonal by parity. It is complete by the uniqueness of the power series expansion. With a simple calculation, we can normalize the basis to the complete orthonormal basis $\left\{1 / \sqrt{2 \pi} z^{j}\right\}_{j=0}^{\infty}$. Thus we see that

$$
S(z, \zeta)=\sum_{j=0}^{\infty} \frac{1}{2 \pi} z^{j} \bar{\zeta}^{j}=\frac{1}{2 \pi} \cdot \frac{1}{1-z \cdot \bar{\zeta}} .
$$

Now it is instructive to write out the Szegö integral for a function in $A(D)$ :

$$
\begin{aligned}
f(z) & =\int_{0}^{2 \pi} f\left(e^{i \theta}\right) \cdot \frac{1}{2 \pi} \cdot \frac{1}{1-z \cdot e^{-i \theta}} d \theta \\
& =\frac{1}{2 \pi i} \int_{0}^{2 \pi} \frac{f\left(e^{i \theta}\right)}{e^{i \theta}-z} \cdot i e^{i \theta} d \theta \\
& =\frac{1}{2 \pi i} \oint_{\partial D} \frac{f(\zeta)}{\zeta-z} d \zeta .
\end{aligned}
$$


Thus we see that the canonical Szegő integral formula is in fact nothing other than the constructive Cauchy integral formula. But only on the disc!

We conclude this section by noting that the integral

$$
\mathbf{S} g(z)=\int_{\partial \Omega} S(z, \zeta) g(\zeta) d \zeta
$$

defines a projection from $L^{2}(\partial \Omega)$ to $H^{2}(\Omega)$. This is because the mapping is selfadjoint, idempotent, and fixes $H^{2}$. We call this mapping the Szegö projection. [Note that the Bergman projection is constructed similarly.]

\section{Constructive Integral Formulas with Holomor- phic Kernel}

In one complex variable it is easy to construct integral formulas. The Cauchy formula is quite trivial to write down. And it is the same for any domain. One may also write down formulas on the ball and polydisc in $\mathbb{C}^{n}$. After that things become complicated. Certainly one should mention here the classic work [HUA] in which the Bergman kernel is calculated for each of the Cartan bounded symmetric domains.

It was not until about 1970 that people found ways to write down integral reproducing formulas with holomorphic kernels in several complex variables. Here we discuss the idea. [It should be noted that both Bungart [BUN] and Gleason [GLE] proved some time ago - by abstract means - that reproducing kernels that are holomorphic in the free variable always exist. But their methods are nonconstructive, and the proofs quite abstract.]

Fix a non-negative integer $k$ and a strongly pseudoconvex domain $\Omega \subset \subset \mathbb{C}^{n}$ with $C^{k+3}$ boundary. Let $\rho: \mathbb{C}^{n} \rightarrow \mathbb{R}$ be a $C^{k+3}$ defining function for $\Omega$ with the property that it is strictly plurisubharmonic in a neighborhood of $\partial \Omega$. It is a standard fact (see [KRA2]), for which we do not provide the details, that the function (known as the Levi polynomial)

$$
L: \mathbb{C}^{n} \times \mathbb{C}^{n} \rightarrow \mathbb{C}
$$

given by

$L_{P}(z)=L(z, P) \equiv \rho(P)+\sum_{j=1}^{n} \frac{\partial \rho}{\partial z_{j}}(P)\left(z_{j}-P_{j}\right)+\frac{1}{2} \sum_{j, k=1}^{n} \frac{\partial^{2} \rho(P)}{\partial z_{j} \partial z_{k}}\left(z_{j}-P_{j}\right)\left(z_{k}-P_{k}\right)$

satisfies the following properties:

(3.1) For each $P \in \mathbb{C}^{n}$, the function $z \mapsto L(z, P)$ is holomorphic (indeed, it is a polynomial);

(3.2) For each $z \in \mathbb{C}^{n}$, the function $P \mapsto L(z, P)$ is $C^{k+1}$;

(3.3) For each $P \in \partial \Omega$, there is a neighborhood $U_{P}$ such that if $z \in \bar{\Omega} \cap\left\{w \in U_{P}: L_{P}(w)=0\right\}$, then $z=P$. 
Our goal is to remove the need to restrict to a small neighborhood of $P \in \partial \Omega$ (property (3.3)) while preserving properties (3.1)-(3.3). We proceed through a sequence of lemmas. Following Henkin, we use the notation

$$
\begin{aligned}
& \Omega_{\delta}=\left\{z \in \mathbb{C}^{n}: \rho(z)<\delta\right\} ; \\
& U_{\delta}=\left\{z \in \mathbb{C}^{n}:|\rho(z)|<\delta\right\}, \quad \delta>0 .
\end{aligned}
$$

Further, let us fix the following constants:

(3.4) Choose $\delta>0$ and $\gamma>0$ such that

$$
\sum_{j, k=1}^{n} \frac{\partial^{2} \rho}{\partial z_{j} \partial \bar{z}_{k}}(P) w_{j} \bar{w}_{k} \geq \gamma|w|^{2}, \quad \text { all } P \in U_{\delta}, \quad \text { all } w \in \mathbb{C}^{n} .
$$

(3.5) Shrinking $\delta$ if necessary, we may select $\kappa>0$ so that

$$
|\operatorname{grad} \rho(z)| \geq \kappa \text { for all } z \in U_{\delta} .
$$

(3.6) With $\delta$ as above, let

$$
K=\sum_{|\alpha|+|\beta| \leq 3}\left\|\left(\frac{\partial}{\partial z}\right)^{\alpha}\left(\frac{\partial}{\partial \bar{z}}\right)^{\beta} \rho(z)\right\|_{L^{\infty}\left(U_{\delta}\right)} .
$$

Lemma 3.7 There is a $\lambda>0$ such that, if $P \in \partial \Omega$ and $|z-P|<\lambda$, then

$$
2 \operatorname{Re} L_{P}(z) \leq \rho(z)-\gamma|z-P|^{2} / 2 .
$$

Proof: Let $\lambda=\frac{1}{2} \gamma /(K+1)$. If $|z-P|<\lambda$, then

$$
\rho(z)=\rho(P)+2 \operatorname{Re} L_{P}(z)+\sum_{j, k=1}^{n} \frac{\partial^{2} \rho}{\partial z_{j} \partial \bar{z}_{k}}(P)\left(z_{j}-P_{j}\right)\left(\bar{z}_{j}-\bar{P}_{k}\right)+R_{P}(z),
$$

where $R_{P}$ is the remainder term for Taylor's formula. Therefore

$$
\begin{aligned}
2 \operatorname{Re} L_{P}(z) & \leq \rho(z)-\sum_{j, k=1}^{n} \frac{\partial^{2} \rho}{\partial z_{j} \partial \bar{z}_{k}}(P)\left(z_{j}-P_{j}\right)\left(\bar{z}_{j}-\bar{P}_{k}\right)+\left|R_{P}(z)\right| \\
& \leq \rho(z)-\gamma|z-P|^{2}+K|z-P|^{3} \\
& \leq \rho(z)-\gamma|z-P|^{2} / 2 \ldots
\end{aligned}
$$

Lemma 3.8 Let $\epsilon=\gamma \lambda^{2} / 20$. If $P \in \partial \Omega, z \in \Omega_{\epsilon}, \lambda / 3 \leq|z-P| \leq 2 \lambda / 3$, then

$$
\operatorname{Re} L_{P}(z)<0 .
$$

Proof: With $z$ as in the hypotheses, we have by Lemma 3.7 that

$$
2 \operatorname{Re} L_{P}(z) \leq \epsilon-\gamma \frac{(\lambda / 3)^{2}}{2}=\gamma \frac{\lambda^{2}}{20}-\gamma \frac{\lambda^{2}}{18}<0 .
$$

We may assume that $\epsilon<\lambda<\delta<1$ (where $\delta$ is as in (3.4) and (3.5)). Let $\eta: \mathbb{R} \rightarrow[0,1]$ be a $C^{\infty}$ function that satisfies $\eta(x)=0$ for $x \geq 2 \lambda / 3$ and $\eta(x)=1$ for $x \leq \lambda / 3$. 
Lemma 3.9 Fix $P \in \partial \Omega$. The $(0,1)$ form

$$
f_{P}(z)=\left\{\begin{array}{lll}
-\bar{\partial}_{z}\{\eta(|z-P|)\} \cdot \log L_{P}(z) & \text { if } & |z-P|<\lambda, z \in \Omega_{\epsilon} \\
0 & \text { if } & |z-P| \geq \lambda, z \in \Omega_{\epsilon}
\end{array}\right.
$$

is well-defined (if we take the principal branch for logarithm) and has $C^{\infty}$ coefficients for $z \in \Omega_{\epsilon}$. If $z$ is fixed, then $f_{P}(z)$ depends $C^{k}$ on $P$. Finally, $\bar{\partial}_{z} f_{P}(z)=0$ on $\Omega_{\epsilon}$. [One may note that this construction is valid even for $P$ sufficiently near $\partial \Omega$.

Proof: On $\operatorname{supp}\{\bar{\partial} \eta(|z-P|)\}$, we have $\lambda / 3 \leq|z-P| \leq 2 \lambda / 3$, so if $z$ is also in $\Omega_{\epsilon}$, then Lemma 3.8 applies and $\operatorname{Re} L_{P}(z)<0$. Therefore $\log L_{P}(z)$ makes sense. It follows that the form has $C^{\infty}$ coefficients for $|z-P|<\lambda$. When $|z-P|>2 \lambda / 3$, we have $\bar{\partial}_{z}\{\eta(|z-P|)\} \equiv 0$, so that $f_{P}(z)$ is smooth. Since $\log L_{P}(z)$ is holomorphic on $\operatorname{supp} \bar{\partial}_{z} \eta(|z-P|)$, it follow that $\bar{\partial} f_{P}(z)=0$ on all of $\Omega_{\epsilon}$. The fact that $f_{P}(z)$ depends $C^{k}$ on $P$ is clear since $L_{P}(z)$ does.

Lemma 3.10 There is a $C^{\infty}$ function $u_{P}$ on $\Omega_{\epsilon}$ such that $\bar{\partial} u_{P}=f_{P}$.

Proof: Since $\epsilon<\delta$, we know that $\rho_{\epsilon}(z) \equiv \rho(z)-\epsilon$ is a defining function for $\Omega_{\epsilon}$; hence $\Omega_{\epsilon}$ is strongly pseudoconvex. By Hörmander's existence theorem for the $\bar{\partial}$ equation (see [KRA2, Ch. 4]), such a function $u_{P}$ must exist.

We now define

$$
\Phi(z, P)=\left\{\begin{array}{lll}
{\left[\exp u_{P}(z)\right] \cdot L_{P}(z)} & \text { if } \quad|z-P|<\lambda / 3 \\
\exp \left[u_{P}(z)+\eta(|z-P|) \log L_{P}(z)\right] & \text { if } \quad \lambda / 3 \leq|z-P|<\lambda \\
\exp \left(u_{P}(z)\right) & \text { if } \quad \lambda \leq|z-P| .
\end{array}\right.
$$

Notice that $\Phi$ is unambiguously defined. To study the properties of $\Phi$, we require two technical lemmas.

Lemma 3.11 If $U ß \mathbb{C}^{n}$ is any open set and $K \subset \subset U$, then any $u \in C^{1}(U)$ satisfies

$$
\sup _{K}|u| \leq C\left(\|u\|_{L^{2}(U)}+\|\bar{\partial} u\|_{L^{\infty}(U)}\right) .
$$

Here the constant $C$ depends on $U$ and $K$ but not on $u$.

Proof: Let $V \subset \subset U$ be a $C^{1}$ domain such that $K \subset \subset V$. Choose $\eta \in C_{c}^{\infty}(V)$ such that $\eta \equiv 1$ on $K$. Apply the full Bochner-Martinelli formula to the function $\eta u$ on $V$. Then the boundary term (the first term of the Bochner-Martinelli formula) vanishes and the desired estimate follows directly from the integrability of the kernel in the remaining term.

The reader who knows something about partial differential equations will note that this result also follows from the uniform ellipticity of the $\bar{\partial}$ operator on compact subsets of $U$. 
Corollary 3.12 Let $\Omega \subset \subset \mathbb{C}^{n}$ be pseudoconvex and $K \subset \subset \Omega$. Let $f$ be a $\bar{\partial}$-closed $(0,1)$ form with $C^{1}$ coefficients. If $u=M f$ is the Hörmander solution to $\bar{\partial} u=f$ (see [KRA2, Ch. 4]), then we have

$$
\|u\|_{L^{\infty}(K)} \leq C\|f\|_{L^{\infty}(\Omega)},
$$

where $C$ depends only on $K$ and $\Omega$ (and not on $f$ or $u$ ).

Proof: We shall not provide the details of this argument, but instead refer the reader to [KRA2, Ch. 5].

Proposition 3.13 Assume once more that $\Omega \subset \subset \mathbb{C}^{n}$ has $C^{k+3}$ boundary. Then $\Phi(\cdot, P)$ is holomorphic on $\Omega_{\epsilon}$. Also there is a $C>0$, independent of $P$, such that for all $z \in \Omega_{\epsilon / 2}$ we have

$$
\begin{aligned}
& \text { if }|z-P|<\lambda / 3, \quad \text { then }|\Phi(z, P)| \geq C\left|L_{P}(z)\right| ; \\
& \text { if }|z-P| \geq \lambda / 3, \text { then }|\Phi(z, P)| \geq C .
\end{aligned}
$$

Proof: If $|z-P| \geq 2 \lambda / 3$, then $\Phi(z, P)=\exp u_{P}(z)$ and $\bar{\partial}_{z} \Phi(z, P)=\left(\exp u_{P}(z)\right)$. $\bar{\partial} u_{P}(z)=\left(\exp u_{P}(z)\right) \cdot f_{P}(z)=0$ by construction. If $\lambda / 3 \leq|z-P|<2 \lambda / 3$ then

$$
\begin{aligned}
& \bar{\partial}_{z} \Phi(z, P)= \exp \left[u_{P}(z)+\eta(|z-P|) \log L_{P}(z)\right] \\
&=\exp \left[u_{P}(z)+\eta(|z-P|) \log L_{P}(z)\right] \\
& \cdot \cdot\left\{f_{P}(z)+\bar{\partial} \eta(|z-P|) \cdot \log L_{P}(z)\right\}
\end{aligned}
$$

since $\log L_{P}(z)$ is holomorphic when $\lambda / 3 \leq|z-P|<2 \lambda / 3$. The last line is 0 by definition of $f_{P}$. The calculation for $|z-P|>\lambda / 3$ is trivial. Hence we find that $\Phi_{P}$ is holomorphic in the $z$ variable, $z \in \Omega_{\epsilon}$.

For the estimate, notice that $f_{P}$ is bounded on $\Omega_{\epsilon}$, uniformly in $P$, so that $u_{P}$ is bounded on $\bar{\Omega}_{\epsilon / 2}$ uniformly in $P$ (by Corollary 3.12 ). So there is a $C^{\prime}>0$ such that $\left|\exp u_{P}(z)\right| \geq C^{\prime}$. Thus

$$
|\Phi(z, P)|=\left|\exp u_{P}(z)\right| \geq C^{\prime} \quad \text { if }|z-P| \geq 2 \lambda / 3
$$

and

$$
|\Phi(z, P)|=\left|\exp u_{P}(z)\right| \cdot\left|L_{P}(z)\right| \geq C^{\prime}\left|L_{P}(z)\right| \quad \text { if }|z-P| \leq \lambda / 3 .
$$

For $\lambda / 3 \leq|z-P| \leq 2 \lambda / 3$, we have by Lemmas 3.7 and 3.8 that

$$
\begin{aligned}
\operatorname{Re} L_{P}(z) & \leq \frac{\epsilon}{2}-\gamma \frac{(\lambda / 3)^{2}}{2} \\
& =\frac{\gamma \lambda^{2}}{40}-\gamma \frac{(\lambda / 3)^{2}}{2} \\
& =-\frac{11 \gamma \lambda^{2}}{360} .
\end{aligned}
$$


Thus

$$
\left|L_{P}(z)\right| \geq \frac{11 \gamma \lambda^{2}}{360} .
$$

We conclude that, for $\lambda / 3 \leq|z-P| \leq 2 \lambda / 3$,

$$
|\Phi(z, P)| \geq\left|\exp u_{P}(z)\right| \cdot\left|L_{P}(z)\right| \geq C^{\prime \prime} \frac{\gamma \lambda^{2}}{360} .
$$

Now we would like to consider the smooth dependence of $\Phi$ on $P$. The subtlety is that our construction of $\Phi_{P}$ involved solving $\bar{\partial} u_{P}=f_{P}$ so, in principle, it appears that we must check the smooth dependence of Hörmander's solution operator on parameters. In fact this type of smooth dependence has been checked for various solutions of the $\bar{\partial}$ problem (see [GRK1]). But, by using a little functional analysis, we may avoid such difficult calculations.

Now fix $z \in \Omega$. Let $\theta \in C_{c}^{\infty}\left(\Omega_{\epsilon}\right)$ satisfy $\theta(z)=1$. Let $s>2 n$. Let $M_{s}^{\theta}$ be the right inverse to $\bar{\partial}_{0,0}$ (the Hörmander solution operator) for the pseudoconvex domain $\Omega_{\epsilon}$. Let notation be as in (3.4) through (3.6). Let $\mu$ be in the dual space of $W^{s}\left(\Omega, \phi_{1}\right)$ [naturally this dual space is just $W^{s}\left(\Omega, \phi_{1}\right)$ itself]. Then

$$
\left\langle\mu, M_{s}^{\theta} f_{P}\right\rangle=\left\langle\left(M_{s}^{\theta}\right)^{*} \mu, f_{P}\right\rangle,
$$

which depends $C^{k}$ on $P$ because $f_{P}$ does.

Proposition 3.14 The function $\Phi(z, P)$ depends in a $C^{k}$ fashion on $P$ for fixed $z \in \Omega_{\epsilon}$.

Proof: Fix $s>2 n$ and let $\mu=e_{z}$ be the point evaluation functional on $C(\Omega) \supseteq W^{s}\left(\Omega, \phi_{1}\right)$. Then, by the preceding discussion,

$$
\left\langle e_{z}, M_{s}^{\theta} f_{P}\right\rangle=\left(M_{s}^{\theta} f_{P}\right)(z)=u_{P}(z)
$$

depends $C^{k}$ on $P$. Therefore $\Phi(z, P)$ itself depends $C^{k}$ on $P$.

Proposition 3.15 Let $\Omega \beta \mathbb{C}^{n}$ be pseudoconvex. Let $\Omega_{k}=\Omega \cap\left\{z \in \mathbb{C}^{n}\right.$ : $\left.z_{1}, \ldots, z_{k}=0\right\}, k=1, \ldots, n$. Let $A_{k}(\Omega)=\left\{f\right.$ holomorphic on $\left.\Omega:\left.f\right|_{\Omega_{k}}=0\right\}$. Then there are linear operators

$$
Q_{i}^{k}: A_{k}(\Omega) \rightarrow\{f \text { holomorphic on } \Omega\} \quad, \quad i=1, \ldots, k,
$$

such that

$$
f(z)=\sum_{i=1}^{k} z_{i} \cdot\left(Q_{i}^{k} f\right)(z)
$$

for all $f \in A_{k}(\Omega)$.

Remark 3.16 We are primarily interested in the proposition when $k=n$. However the proof is by induction on $k$. 
Key in the proof that we are about to present is the following extension result. We cannot provide the detailed proof of this tool, but refer the reader to Section 5.1 of [KRA2].

Theorem 3.17 Let $\Omega \beta \mathbb{C}^{n}$ be pseudoconvex (no assumptions about boundary smoothness, or even boundedness, need be made). Let $\omega=\Omega \cap\left\{\left(z_{1}, \ldots, z_{n}\right)\right.$ : $\left.z_{n}=0\right\}$. Let $f: \omega \rightarrow \mathbb{C}$ satisfy the property that the map

$$
\left(z_{1}, \ldots, z_{n-1}\right) \mapsto f\left(z_{1}, \ldots, z_{n-1}, 0\right)
$$

is holomorphic on $\widetilde{\omega}=\left\{\left(z_{1}, \ldots, z_{n-1}\right) \in \mathbb{C}^{n-1}:\left(z_{1}, \ldots, z_{n-1}, 0\right) \in \omega\right\}$. Then there is a holomorphic $F: \Omega \rightarrow \mathbb{C}$ such that $\left.F\right|_{\omega}=f$. Indeed there is a linear operator

$$
\mathcal{E}_{\omega, \Omega}:\{\text { holomorphic functions on } \omega\} \rightarrow\{\text { holomorphic functions on } \Omega\}
$$

such that $\left.\left(\mathcal{E}_{\omega, \Omega} f\right)\right|_{\omega}=f$. The operator is continuous in the topology of normal convergence.

Proof of the Proposition: If $k=1$ and $n$ is arbitrary then the result follows by setting $Q_{1} f(z)=f(z) / z_{1}$. Now suppose that the result has been proved for $k=K-1$ and for any $n$.

Let $\widetilde{\Omega} \equiv\left\{z: z_{K}=0\right\}$. Let $f \in A_{K}(\Omega)$. Then $\left.\widetilde{f} \equiv f\right|_{\widetilde{\Omega}} \in A_{K-1}(\widetilde{\Omega})$. Therefore, by the inductive hypothesis,

$$
\widetilde{f}(z)=\sum_{i=1}^{K-1} z_{i} \cdot\left(\widetilde{Q}_{i}^{K-1} \widetilde{f}\right)(z) \quad, \quad z \in \widetilde{\Omega},
$$

where $\widetilde{Q}_{i}^{K-1}$ are the operators assumed to exist on $\widetilde{\Omega}$ for $K-1, n-1$.

Now we apply Theorem 3.17. Indeed we let

$$
Q_{K}^{K}(f)(z)=\frac{f(z)-\sum_{i=1}^{K-1} z_{i}\left(\mathcal{E}_{\widetilde{\Omega}, \Omega} \widetilde{Q}_{i}^{K-1} \widetilde{f}(z)\right)}{z_{K}} .
$$

This is well defined and holomorphic on $\Omega$ since the expression in the numerator vanishes on $\widetilde{\Omega}$. Also, let

$$
Q_{i}^{K} f(z)=\mathcal{E}_{\widetilde{\Omega}, \Omega} \widetilde{Q}_{i}^{K-1} \widetilde{f}(z), \quad i=1, \ldots, K-1 .
$$

By algebra, $f(z)=\sum_{i=1}^{K} z_{i} \cdot Q_{i}^{K} f(z)$, all $z \in \Omega$. The induction is now complete.

Corollary 3.18 Let $\Omega \beta \mathbb{C}^{n}$ be pseudoconvex. Then there are continuous linear operators

$$
T_{i}:\{\text { holomorphic functions on } \Omega\} \rightarrow\{\text { holomorphic functions on } \Omega \times \Omega\}
$$


such that, for any holomorphic $f: \Omega \rightarrow \mathbb{C}$, we have

$$
f(z)-f(w)=\sum_{i=1}^{n}\left(z_{i}-w_{i}\right) T_{i} f(z, w), \quad \text { all } z, w \in \Omega .
$$

Proof: Apply Proposition 3.15 to the function $F(z, w)=f(z)-f(w)$ on the domain $\Omega \times \Omega$ with coordinates

$$
\begin{aligned}
z_{1}^{\prime} & =z_{1}-w_{1} \\
& \vdots \\
z_{n}^{\prime} & =z_{n}-w_{n} \\
z_{n+1}^{\prime} & =z_{1}
\end{aligned}
$$

The continuity will follow from the closed graph theorem.

Proposition 3.19 (Hefer's Lemma) Let $\Omega ß \mathbb{C}^{n}$ be strongly pseudoconvex with $C^{4}$ boundary. Let $\Phi: \Omega_{\epsilon / 2} \times \partial \Omega \rightarrow \mathbb{C}$ be the $C^{1}$ singular function constructed above. Then we may write

$$
\Phi(z, \zeta)=\sum_{i=1}^{n}\left(\zeta_{i}-z_{i}\right) \cdot P_{i}(z, \zeta), \quad z \in \Omega_{\epsilon / 2}, \quad \zeta \in \partial \Omega,
$$

where each $P_{i}$ is holomorphic in $z \in \Omega_{\epsilon / 2}$ and $C^{1}$ in $\zeta \in \partial \Omega$.

Proof: Fix $\zeta \in \partial \Omega$. Apply Corollary 3.18 to the function $\Phi_{\zeta}(\cdot)=\Phi(\cdot, \zeta)$ on $\Omega_{\epsilon / 2}$. So

$$
\Phi(z, \zeta)-\Phi(w, \zeta)=\sum_{i=1}^{n}\left(z_{i}-w_{i}\right)\left[\left(T_{i} \Phi_{\zeta}\right)(z, w)\right]
$$

Since this is true for all $w \in \Omega_{\epsilon / 2}$, we may set $w=\zeta \in \partial \Omega$ to obtain

$$
\Phi(z, \zeta)=\sum_{i=1}^{n}\left(z_{i}-\zeta_{i}\right)\left[\left(T_{i} \Phi_{\zeta}\right)(z, \zeta)\right] \equiv \sum_{i=1}^{n}\left(\zeta_{i}-z_{i}\right) P_{i}(z, \zeta) .
$$

It remains to check that $P_{i}$ is $C^{1}$ in $\zeta$. For this, it is enough to verify that $\left(T_{i} \Phi_{\zeta}\right)(z, w)$ is $C^{1}$ in $\zeta$. But, just as in the proof of Proposition 3.14, we let $e_{(z, w)}$ be the point evaluation functional on $\Omega \times \Omega$ and observe that

$$
\left(T_{i} \Phi_{\zeta}\right)(z, w)=\left\langle e_{(z, w)}, T_{i} \Phi_{\zeta}\right\rangle=\left\langle T_{i}^{*} e_{(z, w)}, \Phi_{\zeta}\right\rangle .
$$

The last expression is $C^{1}$ in $\zeta$ by Proposition 3.14 .

We now quickly review the Cauchy-Fantappié formula. See [KRA2, Ch. 1] for the details. 
Theorem 3.20 Let $\Omega \subset \subset \mathbb{C}^{n}$ be a domain with $C^{1}$ boundary. Let $w(z, \zeta)=$ $\left(w_{1}(z, \zeta), \ldots, w_{n}(z, \zeta)\right)$ be a $C^{1}$, vector-valued function on $\bar{\Omega} \times \bar{\Omega} \backslash\{$ diagonal $\}$ that satisfies

$$
\sum_{j=1}^{n} w_{j}(z, \zeta)\left(\zeta_{j}-z_{j}\right) \equiv 1 .
$$

Then, using the notation from Section 2, we have for any $f \in C^{1}(\bar{\Omega}) \cap\{$ holomorphic functions on $\Omega\}$ and any $z \in \Omega$ the formula

$$
f(z)=\frac{1}{n W(n)} \int_{\partial \Omega} f(\zeta) \eta(w) \wedge \omega(\zeta) .
$$

We see that the Cauchy-Fantappié formula is a direct generalization of the Bochner-Martinelli formula discussed above. Now we can give the punchline of this development.

Theorem 3.21 (Henkin [2]) Let $\Omega \beta \mathbb{C}^{n}$ be a strongly pseudoconvex domain with $C^{4}$ boundary. Let $\Phi: \Omega_{\epsilon / 2} \times \partial \Omega \rightarrow \mathbb{C}$ be the Henkin singular function. Define

$$
w_{i}(z, \zeta)=\frac{P_{i}(z, \zeta)}{\Phi(z, \zeta)}, i=1, \ldots, n .
$$

Here $P_{i}(z, \zeta)$ are as in Proposition 3.19. Just as in our earlier discussion, let

$$
\eta(w)=\sum_{i=1}^{n}(-1)^{i+1} w_{i} d w_{1} \wedge \cdots \wedge d w_{i-1} \wedge d w_{i+1} \wedge \cdots \wedge d w_{n}
$$

and

$$
\omega(\zeta)=d \zeta_{1} \wedge \cdots \wedge d \zeta_{n} .
$$

Then, for any $f \in C^{1}(\bar{\Omega}) \cap\{$ holomorphic functions on $\Omega\}$, we have the integral representation

$$
f(z)=\int_{\partial \Omega} f(\zeta) \eta(w) \wedge \omega(\zeta) .
$$

Proof: The functions $w_{i}$ satisfy

$$
\sum_{i=1}^{n} w_{i}(z, \zeta)\left(\zeta_{i}-z_{i}\right) \equiv 1, \quad z \in \Omega, \quad \zeta \in \partial \Omega .
$$

Now apply the Cauchy-Fantappiè formula (see [KRA2, Ch. 5]).

Corollary 3.22 With notation as in the theorem, we have

$$
f(z)=\int_{\partial \Omega} f(\zeta) \frac{K(z, \zeta)}{\Phi^{n}(z, \zeta)} d \sigma(\zeta),
$$

where $K: \Omega_{\epsilon / 2} \times \partial \Omega$ is holomorphic in $z$ and continuous in $\zeta$. In fact, $K(z, \zeta) d \sigma(\zeta)=$ $\eta(z) \wedge \omega(\zeta)$. 
Proof: See [KRA2, Ch. 5].

Of course Corollary 3.22 gives us a constructive integral reproducing formula with kernel that is holomorphic in the free $z$ variable. This is a very useful device, and important for the function theory of several complex variables.

\section{Asymptotic Expansion for the Canonical Ker- nel}

C. Fefferman $[\mathrm{FEF}]$ made an important contribution in 1974 when he produced an asymptotic expansion for the Bergman kernel of a strongly pseudoconvex domain. Basically he was able to write

$$
K(z, \zeta)=P(z, \zeta)+\mathcal{E}(z, \zeta)
$$

where $P$ (the principal term) is, in suitable local coordinates, the Bergman kernel of the ball and $\mathcal{E}$ (the error term) is a term of strictly lower order (in some measurable sense). This powerful formula gives one a means for calculating mapping properties of the Bergman integral. Fefferman himself used the formula to calculate the boundary asymptotics of Bergman metric geodesics (for the purpose of proving the smooth boundary extension of biholomorphic mappings). Fefferman states in his paper - although the details have never been worked out - that there is a similar asymptotic expansion for the Szegö kernel of a strongly pseudoconvex domain.

At about the same time, Boutet de Monvel and Sjöstrand [BOS] used the technique of Fourier integral operators $[\mathrm{HOR}]$ to directly derive an asymptotic expansion for the Szegö kernel of a strongly pseudoconvex domain. This expansion is quite similar to Fefferman's: there is a principal term, which in suitable local coordinates is the Szegö kernel of the ball, and there is an error term which is of lower order. It is not known whether the techniques of [BOS] can be used to derive an asymptotic expansion for the Bergman kernel.

The main purpose of the present paper is to consider another method, due to Kerzman and Stein, for deriving asymptotic expansions for the canonical kernels that is more elementary and uses less machinery. Fefferman's rather complicated argument uses Kohn's solution of the $\bar{\partial}$-Neumann problem as well as the theory of nonisotropic singular integrals. Boutet de Monvel and Sjöstrand's argument uses the theory of Fourier integral operators. The method of Kerzman and Stein [KES] that we treat here uses only basic complex function theory and a little functional analysis.

At this time there are virtually no results about asymptotic expansions for the canonical kernels on weakly pseudoconvex domains. Some interesting partial results appear in [HAN]. But see our Section 6 below. 


\section{The Relation Between Constructive Kernels and Canonical Kernels on Strongly Pseudo- convex Domains}

The ideas that we present now have thus far only been developed on strongly pseudoconvex domains. It is an important open problem to determine how to carry out a similar program on finite type domains or more general domains.

In previous sections, we have defined the Szegő projection $\mathbf{S}: L^{2}(\partial \Omega) \rightarrow$ $H^{2}(\Omega)$. We also have a mapping $\mathbf{H}: L^{2}(\partial \Omega) \rightarrow H^{2}(\Omega)$ that is determined by the Henkin kernel of Corollary 3.22. We note that $\mathbf{H}$ defines a bounded operator from $L^{2}(\partial \Omega)$ to $H^{2}(\Omega)$ (the Hardy space - see [KRA, Chapter 8]) for the following reason.

It is known that $\partial \Omega$, when equipped with balls coming from the complex structure and the usual boundary area measure (see [NSW1], [NSW2]), is a space of homogeneous type in the sense of Coifman and Weiss [COW]. Further, it is straightforward to verify that the Henkin operator $\mathbf{H}$ satisfies the hypotheses of the David-Journé T1 theorem for spaces of homogeneous type (see [CHR] for a nice exposition of these ideas). Thus we may conclude that the Henkin operator maps $L^{2}(\partial \Omega)$ to $L^{2}(\partial \Omega)$. Since the Henkin kernel also obviously maps $L^{2}(\partial \Omega)$ to holomorphic functions, we may conclude that the Henkin integral maps $L^{2}(\partial \Omega)$ to $H^{2}(\Omega)$.

Now of course $\mathbf{S}$, being a projection, is self-adjoint. So $\mathbf{S}=\mathbf{S}^{*}$. It is not at all true that $\mathbf{H}=\mathbf{H}^{*}$, but one may calculate (see below for the details) that $\mathbf{A} \equiv \mathbf{H}^{*}-\mathbf{H}$ is small in a measurable sense.

We also have

$$
\begin{aligned}
\mathbf{H S}=\mathbf{S}, & \mathbf{S H}^{*}=\mathbf{S}, \\
\mathbf{S H}=\mathbf{H}, & \mathbf{H}^{*} \mathbf{S}=\mathbf{H}^{*} .
\end{aligned}
$$

Let us discuss these four identities for a moment.

For the first, notice that $\mathbf{S}$ is the projection onto $H^{2}$, and $\mathbf{H}$ preserves holomorphic functions. So certainly $\mathbf{H S}=\mathbf{S}$. For the second, we calculate that

$$
\left\langle\mathbf{S H}^{*} x, y\right\rangle=\left\langle\mathbf{H}^{*} x, \mathbf{S} y\right\rangle=\langle x, \mathbf{H S} y\rangle=\langle x, \mathbf{S} y\rangle
$$

(because $\mathbf{H}$ preserves holomorphic functions) and thus $=\langle\mathbf{S} x, y\rangle$. Hence $\mathbf{S H}^{*}=$ $\mathbf{S}$. For the third, notice that $\mathbf{H}$ maps to the holomorphic functions, and $\mathbf{S}$ preserves holomorphic functions. And, for the fourth, we calculate that

$$
\left\langle\mathbf{H}^{*} \mathbf{S} x, y\right\rangle=\langle\mathbf{S} x, \mathbf{H} y\rangle=\langle x, \mathbf{S H} y\rangle=\langle x, \mathbf{H} y\rangle=\left\langle\mathbf{H}^{*} x, y\right\rangle .
$$

In conclusion, $\mathbf{H}^{*} \mathbf{S}=\mathbf{H}^{*}$.

Now we see that

$$
\mathbf{S A}=\mathbf{S}\left(\mathbf{H}^{*}-\mathbf{H}\right)=\mathbf{S H}^{*}-\mathbf{S H}=\mathbf{S}-\mathbf{H} .
$$

As a result,

$$
\mathbf{S}=\mathbf{H}+\mathbf{S A}
$$


$\mathrm{SO}$

$$
\mathbf{S}(\mathbf{I}-\mathbf{A})=\mathbf{H}
$$

In conclusion,

$$
\mathbf{S}=\mathbf{H}(\mathbf{I}-\mathbf{A})^{-1}
$$

If indeed we can show that $\mathbf{A}$ is norm small in a suitable sense, then $(\mathbf{I}-\mathbf{A})^{-1}$ is well defined by a Neumann series. Thus we may write

$$
\mathbf{S}=\mathbf{H}+\mathbf{H A}+\mathbf{H A}^{2}+\cdots+\mathbf{H A}^{\mathbf{j}}+\mathbf{H A}^{\mathbf{j}+\mathbf{1}}+\cdots .
$$

Hence we have expressed the Szegö projection $\mathbf{S}$ as an asymptotice expansion in terms of the Henkin projection $\mathbf{H}$. By applying this asymptotic expansion to the Dirac delta mass, this last formula can be translated into saying that the Szegö kernel $S$ can be written as an asymptotic expansion in terms of the Henkin kernel.

It should be noted that Ewa Ligocka [LIG] has shown that these same ideas may be applied to expand the Bergman kernel in an asymptotic expansion in terms of the Henkin kernel. We shall not treat the details of her argument here.

\section{A version of the Kerzman/Stein Theorem on Finite Type Domains}

Here we treat a version of the main result of [KES] on certain finite type domains. Since most of the steps follow [KES] rather closely, we shall outline much of the proof.

We shall concentrate in this section on a convex domain $\Omega=\{z: \rho(z)<0\}$ of finite type in $\mathbb{C}^{2}$ (see [KRA2] for a thorough discussion of the concept of finite type). In particular, the construction of Henkin's reproducing formula with holomorphic kernel as in Section 3 is straightforward for such domains. For one can let $\Phi(z, P)$, for $P \in \partial \Omega$, be given by

$$
\Phi(z, P)=\rho(P)+\sum_{j=1}^{n} \frac{\partial \rho}{\partial \zeta_{j}}(P)\left(z_{j}-P_{j}\right) .
$$

No construction of $\Phi$ using the $\bar{\partial}$ problem, as in Lemmas 3.10 and 3.12 and the discussion adhering thereto, is needed. And we also have no need for the quadratic terms that occur in the Levi polynomial as discussed at the beginning of Section 3.

As a consequence, there is no need for our Lemma 3.7 in this context, and Proposition 3.14 is automatic. Proposition 3.15 and Corollary 3,18 are true for all pseudoconvex domains, and Proposition 3.19 is immediate from our new definition of $\Phi$. Thus we derive a suitable version of Theorem 3.21 and Corollary 3.22 in the present context. We note that Range [RAN1], [RAN2] has explored these ideas on domains of this type. 
Now, looking at [KES], we see that a crucial ingredient is the function $g$ which is defined on page 202. In our context, the function $g$ is simply

$$
g(z, \zeta)=\rho(\zeta)+\sum_{j=1}^{n} \frac{\partial \rho}{\partial z_{j}}(z)\left(z_{j}-\zeta_{j}\right) .
$$

Then the key properties of $g$ enunciated in (2.1.6)-(2.1.10)of [KES] are immediate. Also, for us, the kernel of the operator $\mathbf{A}$ will have the same form as in the paper [KES] of Kerzman and Stein. The only key difference is in the estimate from below on $|g(z, \zeta)|$. Whereas, for Kerzman and Stein, the estimate is

$$
|g(z, \zeta)| \geq C\left[\operatorname{dist}(z, \partial \Omega)+c|\zeta-z|^{2}\right],
$$

for us it is

$$
|g(z, \zeta)| \geq C\left[\operatorname{dist}(z, \partial \Omega)+c|\zeta-z|^{2 m}\right] .
$$

Here $m$ comes from the inequality

$$
\rho(\zeta+\tau) \geq C|\tau|^{2 m}
$$

for $\tau$ a complex tangent vector at $\zeta \in \partial \Omega$. And it is plain that (6.3) holds because the domain $\Omega$ is of finite type in $\mathbb{C}^{2}$. These ideas are discussed in detail in [RAN1] and [RAN2].

It is plain to see that the estimate in (6.2) is not as favorable as the one (6.1) that Kerzman and Stein got to deal with in the strongly pseudoconvex case. But we have the advantage that the boundary of our domain $\Omega$ has a different geometry. For any given boundary point $\zeta \in \partial \Omega$, we have the inequality (6.3), which says that the boundary is $m^{\text {th }}$-order flat at $\zeta$. Thus one can analyze the expressions

$$
\frac{\phi(z)}{[g(\zeta, z)]^{n}}
$$

just as in [KES], and use the methods of [KRA1], to find that the kernel of $H$ is of weak type 1 in the $z$ variable, for each fixed value of $\zeta$. And also of weak type 1 in the $\zeta$ variable, for each fixed value of $z$.

It follows then, because of (2.1.8) and (2.2.2) in [KES], that the kernel of A is in fact of weak type $1+\delta$, some $\delta>0$, in the variable $z$ for each fixed value of $\zeta$. And it is of weak type $1+\delta$ in the variable $\zeta$, for each fixed value of $z$ (it can also be useful here to use nonisotropic polar coordinates-see [FOS]). Thanks to a nice lemma of Folland and Stein (which is presented in detail in [KRA3, Theorem 9.7.7]), we then know that the operator $\mathbf{A}$ maps $L^{p}$ to $L^{p+\epsilon}$, some $\epsilon>0$ (where $\epsilon$ depends in an explicit fashion on $\delta$ ).

But, more importantly, we can make the following analysis. For $\lambda>0$, we can define a fractional differential operator of order $1-\lambda$ by

$$
\mathcal{D}^{\lambda} f(x)=(-\triangle)^{1 / 2}\left(\int \frac{f(t)}{|x-t|^{N-(1-\lambda)}} d t\right) .
$$


Here $\triangle$ is the usual Euclidean Laplacian, and the fractional power is calculated using the Fourier transform. Correspondingly, we let the fractional integration operator of order $\mu$ be given by

$$
\mathcal{I}^{\mu} f(x)=\int \frac{f(t)}{|x-t|^{N-\mu}} d t
$$

Of course, in practice, we would define these operators on a coordinate patch in $\partial \Omega$.

It is easy to check, using elementary Fourier analysis, that $\mathcal{I}^{\mu} \circ \mathcal{D}^{\lambda}=$ id preciseley when $\lambda=\mu$ (and of course $0<\lambda<1,0<\mu<1$ ).

Now we can write

$$
\mathbf{A}=\mathcal{I}^{\lambda} \circ \mathcal{D}^{\lambda} \circ \mathbf{A}=\mathcal{I}^{\lambda} \circ\left(\mathcal{D}^{\lambda} \circ \mathbf{A}\right) .
$$

Here $\lambda$ is chosen to be quite small relative to $\epsilon$. Denote the operator in parentheses on the right of this last formula by $\mathcal{T}^{\lambda}$. So

$$
\mathbf{A}=\mathcal{I}^{\lambda} \circ \mathcal{T}^{\lambda} .
$$

If $\lambda$ is small enough and positive, then the kernel of $\mathcal{T}^{\lambda}$ will be of weak type $1+\delta / 2$ in each variable. So it will certainly map $L^{p}$ at least to $L^{p}$, and in fact to a higher-order Lebesgue space. And certainly $\mathcal{I}^{\lambda}$ will map $L^{2}$ to a Sobolev space of positive order. As a result (and Kerzman/Stein discuss this point in some detail in their paper), by Rellich's lemma, $\mathbf{A}$ is a compact operator on $L^{2}$.

It follows then that the asymptotic expansion of $S$ in terms of $H$ given by formula $(*)$ is a valid, convergent expansion. And that is what we wished to prove.

We conclude by noting that the paper [KES] makes decisive use of the Heisenberg group approximation technique introduced in [FOS] in order to obtain these last results about the mapping properties of $\mathbf{A}$. In the finite type case, one might consider using the ideas in [ROS] to imitate those arguments. We have taken a different approach to the matter in the present paper.

\section{Concluding Remarks}

It is a matter of definite interest to be able to expand the canonical, but nonexplicit, Szegö kernel in terms of a more explicit kernel like the Henkin kernel. Until now, this expansion had only been achieved on strongly pseudoconvex domains. We have indicated here a way to perform the procedure on a class of finite type domains in $\mathbb{C}^{2}$. One might anticipate that the result ought to be true on all finite type domains in any dimension. Of course the analytic difficulties attendant to obtaining such a result are rather formidable.

We hope to explore the matter in future papers. 


\section{REFERENCES}

[ARO] N. Aronszajn, Theory of reproducing kernels, Trans. Am. Math. Soc. 68(1950), 337-404.

[BER] S. Bergman, Über die Entwicklung der harmonischen Funktionen der Ebene und des Raumes nach Orthogonal funktionen, Math. Annalen 96(1922), $237-271$.

[BIN] O. V. Besov, V. P. Ilin, and S. M. Nikol'skii, Inegral Representations of Functions and Imbedding Theorems, V. H. Winston, New York 1978-1979.

[BOS] L. Boutet de Monvel and J. Sjöstrand, Sur la singularité des noyaux de Bergman et Szegö, Soc. Mat. de France Asterisque 34-35(1976), 123-164.

[BUN] L. Bungart, Holomorphic functions with values in locally convex spaces and applications to integral formulas, Trans. Am. Math. Soc. 111(1964), 317-344.

[CHR] M. Christ, Lectures on Singular Integral Operators, CBMS Regional Conference Series in Mathematics, 77, Published for the Conference Board of the Mathematical Sciences, Washington, DC; by the American Mathematical Society, Providence, RI, 1990.

[COW] R. R. Coifman and G. Weiss, Analyse harmonique non-commutative sur certains espaces homogénes, (French) Étude de certaines intégrales singuliéres, Lecture Notes in Mathematics, Vol. 242, Springer-Verlag, BerlinNew York, 1971.

[FEF] C. Fefferman, The Bergman kernel and biholomorphic mappings of pseudoconvex domains, Invent. Math. 26(1974), 1-65.

[FOS] G. B. Folland and E. M. Stein, Estimates for the $\bar{\partial}_{b}$ complex and analysis on the Heisenberg group, Comm. Pure Appl. Math. 27(1974), 429-522.

[GLE] A. Gleason, The abstract theorem of Cauchy-Weil, Pac. J. Math. 12(1962), 511-525.

[GRK1] R. E. Greene and S. G. Krantz, Deformation of complex structures, estimates for the $\bar{\partial}$ equation, and stability of the Bergman kernel, Adv. Math. 43(1982), 1-86.

[HAN] N. Hanges, Explicit formulas for the Szegö kernel for some domains in $\mathbb{C}^{2}$. J. Funct. Anal. 88 (1990), 153-165.

[HEN] G. M. Henkin, Integral representations of functions holomorphic in strictly pseudoconvex domains and some applications, Mat. Sb. 78(120)(1969), 611-632; Math. U.S.S.R. Sb. 7(1969), 597-616. 
[HOR] L. Hörmander, Fourier integral operators. I, —it Acta Math. 127(1971), 79-183.

[HUA] L. K. Hua, Harmonic Analysis of Functions of Several Complex Variables in the Classical Domains, American Mathematical Society, Providence, 1963.

[KES] N. Kerzman and E. M. Stein, The SzegHo kernel in terms of CauchyFantappié kernels, Duke Math. Journal 25(1978), 197-224.

[KRA1] S. G. Krantz, Optimal Lipschitz and $L^{p}$ regularity for the equation $\bar{\partial} u=f$ on strongly pseudo-convex domains, Math. Annalen 219(1976), 233-260.

[KRA2] S. G. Krantz, Function Theory of Several Complex Variables, $2^{\text {nd }}$ ed., American Mathematical Society, Providence, RI, 2001.

[KRA3] S. G. Krantz, Explorations in Harmonic Analysis, with Applications in Complex Function Theory and the Heisenberg Group, Birkhäuser Publishing, Boston, 2009.

[KRA4] S. G. Krantz, Calculation and estimation of the Poisson kernel, J. Math. Anal. Appl. 302(2005)143-148.

[LIG] E. Ligocka, The Hölder continuity of the Bergman projection and proper holomorphic mappings, Studia Math. 80(1984), 89-107.

[NSW1] A. Nagel, E. M. Stein, and S. Wainger, Boundary behavior of functions holomorphic in domains of finite type, Proc. Nat. Acad. Sci. USA 78(1981), 6596-6599.

[NSW2] A. Nagel, E. M. Stein, and S. Wainger, Balls and metrics defined by vector fields, I, Basic properties, Acta Math. 155(1985), 103-147.

[RAN1] R. M. Range, Hölder estimates for $\bar{\partial}$ on convex domains in $\mathbb{C}^{2}$ with real analytic boundary, Several complex variables (Proc. Sympos. Pure Math., Vol. XXX, Part 2, Williams College, Williamstown, Mass., 1975), pp. 31-33. Amer. Math. Soc., Providence, R.I., 1977.

[RAN2] R. M. Range, On Hölder estimates for $\bar{\partial} u=f$ on weakly pseudoconvex domains, Several Complex Variables (Cortona, 1976/1977), pp. 247-267, Scuola Norm. Sup. Pisa, Pisa, 1978.

[ROS] L. P. Rothschild and E. M. Stein, L. P. Rothschild and E. M. Stein Hypoelliptic differential operators and nilpotent groups, Acta Math. 137(1976), 247-320.

[RUD] W. Rudin, Real and Complex Analysis, McGraw-Hill, New York, 1966.

[WEL] R. O. Wells, Differential Analysis on Complex Manifolds, Springer-Verlag, New York, 1979. 
Department of Mathematics

Washington University in St. Louis

St. Louis, Missouri 63130 U.S.A.

sk@math . wustl . edu 